\title{
"etwas über Gleise" oder Versuchsanordnung öffentlicher Platz. Zu Lichtenbergs Sudelbuchaufzeichnung J 528
}

\author{
Sabine Mainberger'
}

\begin{abstract}
The essay analyses the aphorism J 528 in Lichtenberg's Sudelbüchern as a model study of elementary questions and interests of the enlightenment, and as an example of the author's specific way of thinking and writing focusing on three aspects: 1) epistemological: Lichtenberg's text deals with the relationship between reason and anthropological as well as social factors, offering a kind of genealogy of rational behavior; 2) methodological and poetological: The text is a paradigm of Lichtenberg's transferring scientific method to non-scientific subjects; 3) reception: Lichtenberg's poetics find their complement in a specific way of reading: The reader has to be as active as the writer and is invited to do similar scientific experiments as does the observer of the public place he reads about. In other notes in the Sudelbücher, Lichtenberg attends to problems of physiology and psychology of perception as, in the 20th century, they will be a main issue in the theory of Gestalt. As Lichtenberg's way of thinking and writing is a criticism of self imposed immaturity as well as of dogmatic rationalism, enlightenment itself is enlightened.
\end{abstract}

Keywords: Lichtenberg, experiment, scientific method, poetics

Zusammenfassung: Der Aufsatz analysiert die Sudelbuchaufzeichnung J 528 als Modellstudie zu grundsätzlichen aufklärerischen Fragen und als Beispiel für Lichtenbergs besonderes Denk- und Schreibverfahren in drei Hinsichten: 1) in epistemologischer beleuchtet Lichtenbergs Text das Verhältnis der Vernunft zu anthropologischen und sozialen Faktoren und gibt eine Art Genealogie des Rationalen; 2) in methodologischer und poetologischer ist der Text ein Paradigma für den Transfer wissenschaftlicher Methodik auf nichtwissenschaftliche Gegenstände; 3) in rezeptionstheoretischer findet diese Poetik ihr Pendant in einer von Lichtenberg ebenfalls bedachten aktiven, gegenstandgenerierenden Lektüre. In anderen Notaten in den Sudelbüchern ist er wahrnehmungsphysiologischen und -psychologischen Problemen auf der Spur, die die Gestalttheorie des 20. Jahrhunderts beschäftigen werden. Kritisch gegen selbstverschuldete Unmündigkeit wie gegen Rationalismus betreibt er Aufklärung über Aufklärung.

Stichwörter: Lichtenberg, Versuch, wissenschaftliche Methodik, Poetik

\footnotetext{
${ }^{1}$ Professorin für Vergleichende Literaturwissenschaft an der Rheinischen Friedrich-Wilhelms-Universität Bonn. Email: s.mainberger@uni-bonn.de
} 


\section{Mainberger S. - Versuchsanordnung öffentlicher Platz}

Am Ende führt alles auf die Frage hinaus: Entsteht der Gedanke aus Bewegung oder Bewegung aus Gedanke?

Lichtenberg

Vor 20 Jahren wohnte ich einem freien Platz gegenüber, der zwischen 2 parallelen Straßen lag, und nur an der Seite gepflastert war. Ereignete sich nun der Fall daß jemand - doch hier wird es gut sein erst eine Figur zu entwerfen und zwar der Kürze wegen bloß im Kopfe. Man denke sich ein Quadrat, dessen 4 Winkel ich mit A B C D bezeichnen will, und zwar sollen A und B die beiden obern, $\mathrm{C}$ und $\mathrm{D}$ aber die beiden unteren andeuten, und $\mathrm{A}$ soll $\mathrm{D}$, und $\mathrm{B}$ dem $\mathrm{C}$ gegenüber stehn. Ereignete sich, sage ich, der Fall, daß jemand von D nach A oder von $\mathrm{C}$ nach B wollte oder umgekehrt, welches wohl an jedem Tag leicht 500mal geschehen mogte, so wurde es so gehalten. War es schönes Wetter, so ging man so gut man konnte nach der Diagonale. Bei schlechtem Wetter oder wenn der ungepflasterte Teil sehr morastig war wählte man statt der Diagonale die zwei Seiten, wobei gemeiniglich, ehe die Reise angetreten wurde, erst nach dem gegenüberstehenden Winkel hingesehen, und der Schritt etwas beschleunigt wurde. So wie der ungepflasterte Boden mehr abtrocknete fanden sich entweder Kühnere oder solche die ihre Schuhe weniger schonten, und gingen nicht mehr um den ganzen Winkel, sondern kreuzten in Linien über die mit der Diagonale parallel liefen, diese Linien näherten sich nach und nach immer mehr der Diagonale und so ging es mehrenteils. Zuweilen kürzte aber auch [ein] kraftvoller Wanderer, der vor der Stadt schon einen schlechten Weg bestanden hatte, den ganzen Prozeß etwas $\mathrm{ab}$, oder Menschen an deren Schuhen und Strümpfen wenig zu verderben war, oder die weder die einen noch die andern hatten. Die merkwürdigste Erscheinung ereignete sich aber am Morgen wenn des Nachts ein tiefer Schnee gefallen war. So bald es Tag wurde fand ich mehr oder weniger einzelne Punkte die in der Richtung der Diagonale liegen sollten, aber weder darin, noch in irgend einer einfachen Richtung von der ganzen Welt lagen. Sie gehörten öfter einer krummen Linie zu, von der sich 2 gegen eins hätte verwetten lassen, daß sie nicht um 1/8 kleiner als die beiden Seiten des Parallelogramms, aber die ganze Welt gegen einen Groschen verwetten ließ, daß sie nicht um 1/1000 bequemer war (falsch). Um 8 Uhr waren die Punkte schon zu einer Linie verbunden und ehe es eilfe schlug sah man schon sehr gesetzte Männer, die gewiß wußten, daß der kürzeste Weg von einem Winkel eines Parallelogramms nach dem gegenüberstehenden die Diagonale sei, mit stetem und ernstem Tritt durch eine krumme Linie gehen, die vielleicht ein schläfriger Nachtwächter für die Diagonale gehalten hatte. Noch war er schmal, nun begegneten sich aber viele Menschen die gewöhnlich den Pfad so ehrlich teilten daß keiner etwas davon bekam, dadurch wurde er breiter. Damals dachte ich schon etwas über Gleise zu schreiben. ${ }^{2}$

\footnotetext{
${ }^{2}$ LiCHTENBERG 1994 (künftig abgekürzt SB), Erster Band: Sudelbücher I, J 528, 730 f.
} 
Mainberger S. - Versuchsanordnung öffentlicher Platz

Der Experimentalphysiker Lichtenberg studiert nicht nur natürliche, sondern auch soziale Phänomene: In der für das klassische theorein wie fürs moderne Beobachten typischen privilegierten Stellung zum Gegenstand - abseits vom Geschehen, auf erhöhter Warte - hat auch der philosophierende Wissenschaftler Posten bezogen. Was er dort sieht, bringt ihn auf den Gedanken, sich näher mit ,Gleisen` zu befassen.

Vor der Erfindung der Eisenbahn sind Gleise gespurte, technisch oder institutionell vorgegebene Wege. Der Aufklärer Lichtenberg ist indes überzeugt, dass im allgemeinen wie in der aktuellen historischen Lage Erkenntnis und Wissen nur vorankommen, wenn derartige eingefahrene Wege aufgegeben und ganz neue ausprobiert werden. Um der Innovation und Invention willen rechtfertigt, ja, lobt er daher immer wieder digressives Spazieren, Sprünge, Purzelbäume usw. ${ }^{3}$ er weiß um deren heuristisches Potential. Ihn beschäftigt auch nicht nur nebenbei die Frage, unter welchen Bedingungen Menschen bereit sind, Gleise zu verlassen, oder diese eine dirigierende Macht ausüben. Die Frage ist vielmehr eine andere Variante der großen jener Zeit: der nach dem Weg aus selbstverschuldeter Unmündigkeit. Im Überqueren des Platzes scheint sich dieses grundsätzliche Problem in konzentrierter Form darzubieten. An ihm mag sich als einem Modellfall studieren lassen, wann sich Rationalität in der Praxis durchsetzt und was sie behindert, darüber hinaus aber auch - und das ist die interessantere Dimension -, was das Widerspiel von sozialem Verhalten und Vernunft, deren Imago die Geometrie ist, für die aufklärerische Vorstellung eines vernunftgeleiteten Lebens bedeutet. Denn nicht nur verunreinigen oder stören die Praktiken eine ideale Vernunft, wie es ein Denken in topischen Bahnen will, vielmehr scheinen die Parameter derartiger Topoi hier in Frage gestellt.

\footnotetext{
${ }^{3}$ Vgl.v.a.: ,[...] man muß notwendig heut zu Tage anfangen, auch bei den ausgemachtesten Dingen, oder denen wenigstens, die es zu sein scheinen, ganz neue Wege zu versuchen. Die Gleise oder vielmehr die gebahnten Wege sind etwas sehr Gutes, - aber wenn niemand nebenher spazieren wollte, so würden wir wenig von der Welt kennen. Die Leute die in der Gegend wohnen, das ist, die, die sich in der Welt nur einem kleinen Fach widmen, müssen alles versuchen. Der Reisende bleibt auf der Heerstraße, der Gutsbesitzer muß alle Stellen untersuchen.” K 312, SB II, 455. Vgl. z.B. auch: „Wir sind auf dem Wege zur Untersuchung der Natur in ein so tiefes Geleise hinein geraten, daß wir immer anderen nachfahren. Wir müssen suchen herauszukommen." K 306, SB II, 453. Oder: „Die Lehre von der Elektrizität ist jetzt da, wo man gewöhnlich passiert, so abgetreten und abgesucht, daß an der Heerstraße nichts mehr zu gewinnen ist; man muß querfeldein marschieren, und über die Gräben setzen. Diese Methode, die man wohl die unmethodische nennen könnte, ist überhaupt nebenher sehr zu empfehlen." K 384, SB II 472. Vgl. auch J 1331, SB 245, J 1602 und 1603, SB II, 294 f., J 1990, SB II,357 ff., und die Hinweise zu J 528 im Kommentarband zu SB I und II, 590 f.
} 
Mainberger S. - Versuchsanordnung öffentlicher Platz

Eine ,Modellstudie‘ hat Lichtenberg offenbar mit dem zitierten Text vor, denn er hat ihn wohl für eine geplante Publikation vielfach korrigiert. ${ }^{4}$ Das Vorhaben blieb unausgeführt, aber die Aufzeichnung bietet, zumal in Verbindung mit anderen in den Sudelbüchern, genug, um Gewicht und theoretisches Potential des Textes zu erfassen. Ich möchte ihn im folgenden in mehreren Hinsichten als Modell lesen:

In epistemologischer Hinsicht: Das beschriebene Geschehen ist Paradigma dafür, wie anthropologische und soziale Faktoren Vernunft ebenso bedingen wie deren Gegenteil; die Erzählung von der ,ergangenen` Geometrie ist eine Art Genealogie des Rationalen.

In methodologischer und poetologischer Hinsicht: Der Text ist ein eindrucksvolles Beispiel dafür, wie Lichtenberg seine wissenschaftliche Beobachtungs- und Versuchspraxis auf nicht-physikalische Probleme überträgt und aus einem Gegenstand der alltäglichen Erfahrung, der Erzählung und Beschreibung einen der wissenschaftlichen Befragung und Beobachtung macht. Dieser Transfer bildet eine wesentliche Komponente in Lichtenbergs schriftstellerischer Praxis: Die Methodik des Experimentalphysikers wird Poetik.

In rezeptionstheoretischer Hinsicht: Der Leser befindet sich dem Erzähler und Beobachter im Text gegenüber in einer ähnlichen Situation wie dieser gegenüber den Passanten auf dem Platz, und zugleich liegt darin eine Potenzierung: Er beobachtet den Beobachter, und wie dieser ist er auch in die Konstitution des Objekts involviert. Die Lektüre selbst ist aktiv und gegenstandsgenerierend. Als solche bildet sie das spezifische, der Poetik der Sudelbücher entsprechende Pendant auf der Seite der Rezeption.

Die drei Hinsichten lassen sich allerdings nur in abstracto so schematisch trennen und nebeneinanderstellen.

1) Die Fußgänger auf jenem Göttinger Platz verhalten sich der gebotenen Beschreibung nach so, dass sich an ihnen grundsätzliche Fragen studieren lassen. Bei schönem Wetter folgt niemand den ,Gleisen', d.h. den gepflasterten Wegen an den Seiten, sondern alle nehmen die Diagonale. Für den Beobachter ist diese Wahl des Weges selbstverständlich, und nur die Abweichungen davon gelten ihm als erklärungsbedürftig. Das heißt - und damit sei schon eine erste Bemerkung zur Methodik eingeschaltet -, es gibt für ihn einen unbefragt

\footnotetext{
${ }^{4}$ Vgl. Kommentarband zu SB I und II, $590 \mathrm{f}$.
} 
Mainberger S. - Versuchsanordnung öffentlicher Platz

vorausgesetzten Standard der Vernunft: den der geometrischen, und Modell des Normalmenschen ist ebenso unbefragt der homo oeconomicus, der auf kürzest möglichem Weg zum Ziel kommen möchte. Diese Annahme schließt bereits einiges aus: den verträumten Spaziergänger etwa, der gern einen Umweg macht, oder den geselligen Kleinstadtbewohner, der mit jemanden plaudern möchte und dies auf dem gepflasterten Stück sicher bequemer tun kann als mitten auf dem Platz. Aber wie der Experimentalphysiker bei einem Versuch die Zahl der Parameter einschränken muß, um zu einer gültigen Aussage zu gelangen, so reduziert er auch diesen Gegenstand aus der, Sozial-Physik': Die hier zu vergleichenden vier Möglichkeiten sind die Situationen bei schönem Wetter, bei Regen, nach dem Regen und bei frischem Schnee.

Im ersten Fall, bei gutem Wetter, verlassen alle die Gleise, bei schlechtem folgen sie ihnen. Die Lage dazwischen - allmähliches Trocknen des Bodens - lässt dagegen eine Reihe von Varianten entstehen: Kompromisse zwischen den Alternativen Folgen und Verlassen der Gleise, zwischen dem zeitaufwendigeren Pflaster und der zeitsparenden Diagonale durch den Morast: das Schneiden der Ecken bzw. die ,Graduierung' der Diagonale. Die Passanten halten sich dabei in jedem Fall an geometrische Formen, die Diagonale im strikten Sinn bringen sie indes nur verzögert zur Geltung. Die Abweichung davon oder ihre Durchsetzung hängt von sozialen und ökonomischen Faktoren ab, die sich im jeweiligen Verhältnis der Passanten zum Gehen und zu ihren Schuhen ausdrücken: Die einen nehmen Rücksicht auf ihre Schuhe, bei anderen kommt es nicht mehr darauf an, weil sie schon lange unterwegs sind, wieder andere haben überhaupt keine. Wo sonst einheitlich geometrische Vernunft herrscht (oder der Nutzen der Institution, des Pflasters gilt), modifizieren die sozial-ökonomischen Unterschiede die Praxis der Platzüberquerung. ${ }^{5}$

\footnotetext{
${ }^{5}$ Die Modifikation folgt einem dichotomischen Muster: gute Schuhe - im Gleis gehen, schlechte/keine Schuhe Gleis verlassen. Überhaupt ist in dem ganzen Text ein Kombinationsschema am Werk, das man auch tabellenförmig aufzeichnen könnte. Vier Elemente werden miteinander verknüpft: gutes Wetter - kurzer Weg (= Diagonale); schlechtes Wetter - langer Weg (= an den Seiten entlang); gutes Wetter - langer Weg (als irrelevant ausgeschieden); schlechtes Wetter - kurzer Weg, aber - und das ist das Problem - ,kurzer Weg = Diagonale“ trifft in diesem Fall nicht zu. Was aber steht dann an der Stelle dieses Elements? Wenn die gepflasterten Strecken die Gleise sind, kann das Schema auch so beschrieben werden: gutes Wetter - Gleis verlassen; schlechtes Wetter - im Gleis gehen; gutes Wetter - im Gleis gehen (als irrelevant ausgeschieden); schlechtes Wetter - Gleis verlassen, aber - und das ist das aufzuklärende Irreguläre - zugleich auch im Gleis gehen! Die Situation nach dem Regen, bei allmählichem Trocknen des Bodens, kann man selbst als eine Kombination ansehen, nämlich als eine von gutem und schlechtem Wetter: Bei gut-schlechtem Wetter geht man dementsprechend einen kurz-langen Weg, die (alten) Gleise werden verlassen und zugleich (neue) verfolgt; es ist ein Gehen-ohne-und-im-Gleis. Im Rahmen von Geometrie und Kombinatorik sind ,kurz-lang' und ,ohne-und-
} 
Mainberger S. - Versuchsanordnung öffentlicher Platz

Der Schnee dagegen tilgt alle Differenzen, die straßenbaulichen und die gesellschaftlichen: Das Pflaster ist in diesem Fall kein Vorteil mehr, es ist genauso bedeckt wie die Zone in der Mitte; Träger guter Schuhe (die ,gesetzten Männer') und solche schlechter nehmen alternativlos alle den gleichen Weg. Der Schnee hat zunächst den ganzen Platz in eine zwischen den Ecken ausgespannte entstrukturierte weiße Fläche verwandelt. Sie ähnelt dem Blatt Papier, und daher scheint nichts näher zu liegen, als die Gesetze der Geometrie hier umstandslos zu praktizieren. Diese Möglichkeit wird indes von einem anderen Faktor durchkreuzt, der ein sozialer ist, aber über die Stände hinweggeht: vom Herdentrieb und von der Selbsttäuschung. ${ }^{6}$ Wie bei schönem Wetter folgt auch in dieser Situation niemand den Seiten, alle wollen abkürzen und suchen daher nicht die verdeckten Gleise auf, aber statt die Diagonale einzuschlagen, gehen alle in der zufällig vom ersten Passanten, dem Nachtwächter, gespurten Bahn. Dass diese vom Weg der Vernunft abweicht, liegt an dessen Übermüdung; dass aber niemand sie korrigiert, hat ausdrücklich andere Gründe als die der Zeit- und der Kräfteökonomie. Wäre jener bei wachen Sinnen durch die Diagonale gegangen, dann ließen sich allerdings Rationalität und Herdentrieb gar nicht unterscheiden. Die situative Schwäche seiner Vernunft ist somit von wissenschaftlichem Nutzen. Im Fall des Schlamms sucht jedes Individuum sich seinen eigenen Weg und verhilft dabei nach Maßgabe seiner Kräfte der Rationalität zum Durchbruch, denn im Morast gibt es keine Spuren. Der Schnee dagegen hat erst die sonst geltenden Marken getilgt und fungiert dann selbst als Gedächtnisspeicher: Die erste Spur bleibt, und sie wird zum Gleis für alle. Nicht die Institution, sondern der Vorgänger im wörtlichsten Sinne schafft die verbindliche Bahn; die übrigen sind Nachfolger, die sich ans Gegebene und auf sie Gekommene halten, d.h. Traditionalisten. Die Rationalität ist in der krummen Linie suspendiert, denn diese stellt keinen Kompromiss und keine verzögerte geometrische Vernunft dar, sondern eine Form jenseits aller Geometrie. Sie ist die Bahn der ,schläfrigen“ und weiter nicht zum Erwachen kommenden Vernunft: nicht die Spur des Selbstdenkens und der Aufklärung, sondern der

im-Gleis‘ paradox oder unmöglich; sollen sie einen Sinn haben, muß man diesen Rahmen wieder verlassen, d.h. wieder auf den der Beobachtung eines sozialen Phänomens zurückkommen. Lichtenbergs Überlegungen pendeln hin und her zwischen den beiden Arten der Betrachtung: Die erstere konstruiert das Problem ,Missverhältnis zwischen Wissen und Verhalten, Ratio und Praxis'; die letztere beantwortet nicht die Frage, wie es dazu kommt, zu vermeiden wäre o.ä., sondern lässt etwas hervortreten, das die Problemstellung selbst fraglich macht.

${ }^{6}$ Vgl. den vorletzten Satz von J 528. In Lichtenbergs Beschreibung haben die Menschen gar nichts davon, dass sie der Spur folgen: Der Weg wird nur breiter, aber nicht kürzer. Was meint indes der Klammer-Zusatz „falsch“ zur Behauptung, der Weg sei durchaus nicht bequemer? 
Mainberger S. - Versuchsanordnung öffentlicher Platz

Benommenheit, des Dämmers bestenfalls und der epistemischen Nachtwächterei. Ihre Kurven und Knicke bilden ein negatives Seitenstück zu allen hier möglichen Bahnen: zur rationalen Diagonale wie zu jeder anderen Linie eines sich dem ,Gleis ${ }^{6}$ entziehenden Denkens ${ }^{7}$ (und natürlich auch zu den institutionalisierten Wegen des Carrés). Insofern bietet diese Situation tatsächlich ein eindrucksvolles Bild für den Stand der Vernunft und kann, wie Lichtenberg formuliert, als „heuristische[s] Hebezeug[]”8 für Menschen und Gesellschaft betreffende Betrachtungen verschiedenster Art dienen. Die alltägliche Szene wird zum Denkbild.

2) Der Text zeigt den methodischen Zugriff des Wissenschaftlers Lichtenberg auf seinen Gegenstand und genauer die Art, wie er einen solchen überhaupt hervorbringt. Von anderen beobachteten Bewegungsbahnen, die vor den Augen des Betrachters entstehen, wie etwa vor denen eines Zuschauers beim Tanz (Vgl. z. B. MAINBERGER 2005), unterscheiden sich diese Bahnen hier dadurch, dass nicht der Beobachter ihren Gang durch den Raum aufzeichnet, sondern dass die Akteure selbst es tun. Ihre Linien auf dem Boden sind insofern Linien oder ,Graphen“ besonderer Art, nämlich Selbstregistrierungen. Sie sind die Spuren eines raumzeitlichen Geschehens - eine spezifisch reduzierte Fixierung und Visualisierung dessen, was sich sonst dem Auge entzieht. Das teilen sie mit Lichtenbergs Staubfiguren auf der elektrisierten Unterlage. ${ }^{9}$ Anders als bei diesen aufzeichnungstechnischen Unternehmen aber handelt es sich hier nicht um ein artifizielles Arrangement zur Erzeugung von Spuren: Es gibt keine eigens geschaffene Versuchsanordnung und keine Maßnahmen an den Akteuren oder im Raum zwischen ihnen und dem Beobachter. Die einzige Aktivität des Analytikers scheint tatsächlich die der Beobachtung zu sein; alles Übrige tun die Gehenden selbst, ohne

\footnotetext{
${ }^{7}$ Aus Wittgensteins Sicht wären die unbegründbaren, kontingenten Bahnen, die aber verbindliche Konventionen darstellen, ein Analogon zu Funktionsweisen der Sprache. Statt diese an einem idealen Maß korrigieren zu wollen, muss der Philosoph die Sprachspiele beobachten und beschreiben: Sie sind die (tatsächlich gegangenen, unidealen) Bahnen der Sprache, deren ,Gleise', die in all ihrer ,Krummheit' doch das Erreichen des kommunikativen Ziels erlauben. Die skeptische Philosophietradition betrachtet auch Fragen der Moral derart: Da Probleme auf diesem Gebiet nicht dogmatisch-doktrinär zu lösen sind, hält sich der (pyrrhonische) Skeptiker undogmatisch an die altägliche Lebenserfahrung. Descartes versucht entsprechend das Denken, nicht aber die Moral radikal neu zu begründen. Lichtenbergs Haltung hat etwas vom doktrinären Aufklärer und etwas vom Rationalismus-Skeptiker: Sie wendet sich gegen das Halbwissen, das die geometrische Vernunft verfehlt, und ist, jenseits der Mathematik, einsichtig in die Bedingtheit, die nicht-ideale Herkunft der Vernunft.

${ }^{8}$ (K 312, SB II, 455)

${ }^{9}$ Die erst zufällig, dann experimentell erzeugten Erscheinungen lässt er in einer Art Druckverfahren sich selbst aufzeichnen. Vgl. Von einer neuen Art, die Natur und Bewegung der elektrischen Materie zu erforschen, SB III, (24-34) 25 f. Vgl. dazu z.B. auch GAMPER 2009: 359-385, v.a. 373-379.
} 
Mainberger S. - Versuchsanordnung öffentlicher Platz

Aufforderung, Absprache und Plan. Die Bedingungen, unter denen ihre Aktionen stattfinden, lassen sich ihrerseits nur konstatieren, aber nicht beeinflussen. Da die Vorgänge sich nicht als singuläre und zufällige Erscheinungen darbieten, sondern sich wiederholen (,an jedem Tag leicht 500mal”), bilden sie auch gewissermaßen von sich aus die nötigen Vergleichsreihen für repräsentative Ergebnisse. Der Platz scheint so das Paradox einer natürlichen Experimentalanordnung zu sein; den sichtbaren Bahnen eignet als ,natürlichen Zeichen', Spuren, Indices, in die keine Inskriptionstechnik eingreift, das Pathos der vera ikon; die Gesetze der Vorgänge brauchen hier offenbar nur noch abgelesen zu werden.

Genauer besehen aber verkürzte diese Auffassung die Lage entscheidend, weil sie die Rolle des Beobachters aussparte. Erst sein Blick vom Fenster aus macht nämlich aus der Lebenswelt ein wissenschaftliches Tableau, aus dem städtischen Platz eine Versuchsanordnung. Und wie im Labor, so kommen auch hier eine ganze Reihe von Faktoren zusammen, materielle und theoretische, die zeigen, dass es sich hier nur vermeintlich um ,Selbstregistrierung' handelt:

Die menschliche Natur zeichnet mitnichten ohne Zutun ihre Bewegungen auf, auch wenn das Eingreifen des Beobachters nicht unmittelbar sichtbar wird, weil es sich nicht in handgreiflichen Vorrichtungen und Apparaten verkörpert. Seine Intervention lässt sich jedoch durchaus mit hinreichender Genauigkeit angeben: Der Wissenschaftler nimmt eine bestimmte, beobachtende Haltung an einem bestimmten Ort ein. Das Fenster liegt über dem Niveau des Platzes, denn nur von höherer Warte lassen sich die Längen der Wege an der Seite und durch den Trampelpfad abschätzen. Und er sieht dem Geschehen auf andere Weise zu als einer, der sich zwischendurch von seinem Buch erholt, oder als einer, der hofft, von Vorgängen draußen aus seiner Langeweile gerissen zu werden. Er abstrahiert z.B. von seinem Verständnis für das, was die Menschen tun (könnte er nicht von seinem Fensterplatz aus die Längen der zurückzulegenden Strecken vergleichen, dann würde er selbst auch in der krummen Linie gehen); er konstruiert die Variablen und ihre Kombinationsmöglichkeiten, hält Gesehenes fest, in diesem Fall nur verbal, und transformiert für das imaginäre Auge des Lesers den Platz in eine geometrische Form: „Man denke sich ein Quadrat...”

Ein scheinbar ,bloßes Beobachten“ übersetzt das Sichtbare auf entscheidende Weise; der Beobachter investiert einen spezifischen Habitus und vorgängige Theorie in den Gegenstand: Der Platz mit den Straßen ist unter seinem Blick nicht länger ein Ort des städtischen Verkehrs, sondern eine eigens entworfene ,Figur', und seine Überquerung keine 
Mainberger S. - Versuchsanordnung öffentlicher Platz

alltagspraktische Handlung, sondern ein unter bestimmten gegebenen Bedingungen gestelltes Problem: „Ereignete sich nun der Fall, daß jemand ...”. Der Gang über den Platz wird qua Beobachtereinstellung ein wissenschaftlicher Gegenstand. Erst im Rahmen dieser Transformation aber erscheint das zu Sehende ,merkwürdig', nämlich als Kontrast zwischen einer Idealität und einer ihr nicht gemäßen Praxis. Der Kontrast selbst ist indes kein ,natürlicher', sondern das Ergebnis einer artifiziellen Anordnung, in die die verschiedenen Komponenten der Szene gebracht sind. Und der so konstruierte Kontrast ist erst die Frage, auf den die Beschreibung der ,Fälle‘ eine implizite Antwort gibt: Im ersten Fall (demjenigen nach dem Regen) geht aus einer unidealen Praxis, genauer aus Akten partieller, situativer, gradueller Vernunft und ,Kühnheit', d.h. der Abweichung vom Gleis, in einem kollektiven Prozess allmählich die ideale Diagonale hervor; dieser ,Fall‘ antwortet auf das Problem des Kontrasts oder Mißverhältnisses von Vernunft und Praxis mit einer Genealogie der ersteren und einer optimistischen Fortschrittsgeschichte. Der zweite Fall (derjenige bei Schnee) stellt das Gegenteil dazu dar: Die krumme Linie konterkariert die alten, bequemen Gleise (die Wege an den Seiten) und die neue, von Gleisen sich lösende Bahn (die Diagonale), sie ist weder bequem noch intelligent - und etabliert sich dennoch als neues Gleis.

In welchem Maße der Wissenschaftler seinen Gegenstand schafft und mit seinem Tun in das zu Beobachtende involviert ist, macht Lichtenberg hier nicht eigens zum Thema. Aber er lässt den Leser an der Erschaffung des wissenschaftlichen Objekts teilhaben: Wie die Spuren im Schnee und die Diagonale durch den Morast vor den Augen des Beobachters entstehen, als seien sie für ihn und seine Tätigkeit gemacht, so wird auch die ganze Szene als kunstvoll arrangierte vor das innere Auge des Lesers gerufen; er sieht sie nicht einfach vor sich, sondern sieht ihrer Inszenierung zu: Der Text beginnt erzählend und scheint dem Leser jenen für das Schreiben dieser Zeit so charakteristischen autobiographischen Pakt anzubieten: „Vor 20 Jahren wohnte ich einem freien Platze gegenüber...” Der angeschlagene narrative Duktus weicht indes gleich wieder einem anderen. Der Erzähler unterbricht sich, scheinbar spontan einer Eingebung des Augenblicks folgend, schon mitten im zweiten Satz, um eine wissenschaftliche Denk- und Redeweise zu empfehlen: „,- doch hier wird es gut sein erst eine Figur zu entwerfen...”. Als geschähe es aus Rücksicht auf den Leser und im Konsens mit ihm, wechselt das rhetorische Register vom narrativen Imperfekt und der ersten Person zum unpersönlichen konjunktivischen Imperativ, wie er für Aufgabenstellungen in der Mathematik typisch ist: „Man denke sich ein Quadrat...”. Hier leitet diese Wendung ein imaginäres bzw. 


\section{Mainberger S. - Versuchsanordnung öffentlicher Platz}

ein Gedankenexperiment ein, einen Versuch „bloß im Kopfe“. Dialogisch und performativ, in kunstvoller Mimesis eines lebendigen Austauschs mit dem Leser, findet auf diese Weise vor dessen Augen die Transformation eines erinnerten Geschehens in eine wissenschaftliche Modellsituation statt, in der verschiedene Fälle durchgespielt werden. ${ }^{10}$ Und mit ihm wandelt sich der Erzähler zum Beobachter, der nun nicht mehr persönlich Erlebtes und zufällig Bezeugtes mitteilt, sondern von wiederholten Vorgängen berichtet, die sich zu anderer Zeit und an anderem Ort ebenfalls ereignen und die sogar - das ist die Voraussetzung für wissenschaftliche Versuche - gezielt wiederholt werden könnten. Die noch zum Erzählen des einst Gesehenen gehörige Formulierung „Ereignete sich nun der Fall, daß jemand_..” schlägt mit ihrer Kombination von narrativem Tempus und technischem Vokabular gewissermaßen die Brücke zum wissenschaftlichen Diskurs und wird nach der Unterbrechung zur Einführung der imaginierten geometrischen Figur wieder aufgegriffen. („Ereignete sich, sage ich, der Fall...”) Am Ende mündet die Präsentation des Problems erneut in die Narration: „Damals dachte ich schon etwas über Gleise zu schreiben.” Die Erzählung bildet also den Rahmen für das Experiment, zu dessen Beobachtung der Leser hier eingeladen wird wie ein Gast ins Labor oder wie der Laie zur Vorführung eines physikalischen Experiments. Diese fanden zu Lichtenbergs Zeit als spektakuläre Vorführungen statt; ihr Sinn lag eher im Erstaunen und in der Unterhaltung als in der Erkenntnis. ${ }^{11}$ Hier wird dem Zuschauer ausdrücklich ein ,merkwürdiges‘ Schauspiel geboten, die Auswertung des Gesehenen ihm aber vorenthalten. Er mag es daher beim wunderlichen Erlebnis belassen oder aber, und das dürfte im Sinn des einladenden Wissenschaftlers liegen, sie selbst vornehmen und sich dabei auch Gedanken darüber machen, wie sehr der Zuschauer selbst Mitakteur bei diesem wissenschaftlichen Theater ist.

Um ein derartiges Mitspielen und Mitwirken an der Erzeugung des zu beobachtenden Gegenstandes geht es bei Lichtenberg immer wieder. Denn Beobachten ist, wie er bemerkt und wachsam verfolgt, kein passives Registrieren von Gegebenem, sondern eine transformierende und generierende Aktivität. In diesem Sinn ist es in der bekannten

\footnotetext{
${ }^{10}$ Bei dem zu Erzählenden handelt es sich freilich auch nicht um singuläre Ereignisse, wie es das Narrativ in der Regel fordert, sondern um wiederholte, um Gewohnheiten und Habitus - im Sinne Genettes um den iterativen Typ des Erzählens, der dem Beschreiben nahe steht. Der Sprung zwischen diesem Erzählen und der Schematisierung des Beobachteten, wie sie Lichtenberg hier vornimmt, ist daher weniger groß.

11 Vgl. z.B. HANKINS 1985: 53ff., ARBURG 1998: 49f., vgl. auch GAMPER 2009 und die dort angegebene Literatur.
} 


\section{Mainberger S. - Versuchsanordnung öffentlicher Platz}

Aufzeichnung über das ordnende Sehen im Muster des Betthimmels ${ }^{12}$ selbst Gegenstand des Interesses. Wie die Platzüberquerungen, so soll auch das Mustersehen möglicher Ausgangspunkt zu weiterreichenden Überlegungen sein; auch an dieses scheinbar marginale Phänomen sollen sich philosophische Gedanken anschließen lassen: „Ich glaube [die] Sache könnte auf höhere Dinge angewendet werden.” Die projektive, gestaltende Wahrnehmung „Bilder in den Wolken und in bunten Steinen” - interessiert, wo es um das inventive Ähnlichkeiten-Finden und Relationieren geht. Lichtenberg akzentuiert daran allerdings nicht die Kreativitätsübung, wie sie seit Leonardo topisch ist und $\mathrm{zu}$ seiner Zeit etwa, mit spezifischen Abwandlungen und Begründungen, von dem englischen Maler Alexander Cozens im Maluntericht gelehrt wird. ${ }^{13}$ Die Aufmerksamkeit gilt bei Lichtenberg nicht dem Entfesseln von Phantasie und sowieso nicht der Technik, ein Landschaftsbild im Großen zu strukturieren, damit sich der Maler nicht in die Einzelheiten verliert; es gilt vielmehr dem Verfahren und der prinzipiellen Möglichkeit, ,in der größten Unordnung Ordnung [zu] sehn”: Denn „so wie ich nur einen Rhombus von etwa einem Quadratzolle, oder von 4 oder von 9 usw. Quadratzollen recht deutlich ins Auge faßte, so verwandelte sich für mein Auge sogleich die ganze Fläche in solche Rhombos, alle von der Größe des angenommenen. [...] Wenn ich ein Neues [Muster, SM] versuchte, so hielt es immer anfangs etwas schwer, war es aber im Gange, so war auf einmal das Ganze wie plötzlich kristallisiert. [...] In einer Menge gleichförmig verteilter Punkte könnte ich allerlei Zeichnungen sehen und allerlei Muster, die an einem Ende der Fläche erst gehörig gefaßt sich bald auch im übrigen finden würden.” Aktives Sehen heißt hier das Amorphe strukturieren durch die Projektion eines Schemas: eine Spur legen, ein Gleis. „Muster” entstehen so „aus objektiven und subjektiven Anlagen zugleich”. Eine berühmte Formulierung Lichtenbergs variierend, könnte man auch sagen, sie entstehen aus einem ,ich sehe“ und einem, es sieht'. Dabei aber hat der Betrachter die Möglichkeit, die Muster zu variieren: Sie sind zwar Gleise der Wahrnehmung, aber sie

\footnotetext{
12 Vgl. J 532, SB I, 732 (die im Text folgenden Zitate jeweils daraus). Der Kommentar bringt diese Aufzeichnung und $\mathbf{J} 528$ auch in Beziehung; beide dürften demnach auf dem Krankenlager entstanden sein. Für J 528 hieße das: Die Verwandlung der Konstellation von Fenster und Platz in eine Versuchsanordnung mit Beobachter geschieht retrospektiv, in der Erinnerung an die sich wiederholende Szenerie. Erst aus dieser distanzierten Sicht, anhand einer Gedächtnis-Aufzeichnung, und fern vom tatsächlichen Ort des Geschehens wie des Beobachtens (nicht zuletzt in einer bemerkenswert anderen Lage im wörtlichen Sinn, nämlich liegend) wird aus dem alltäglichen Geschehen der wissenschaftliche Gegenstand, aus dem oft Bemerkten und Gewöhnlichen etwas Neues. In Analogie zum Muster im Betthimmel kann man sagen: Die gleichen Komponenten werden in anderer Situation anders zusammengesetzt, ein neues Muster wird wahrgenommen.

${ }^{13}$ Vgl. z. B. LebENSZTEJN 1990, und BUSCH 1993: v.a. 344-353.
} 
Mainberger S. - Versuchsanordnung öffentlicher Platz

bannen diese nicht. Nicht nur sehen verschiedene Personen in irregulären Flecken oder regelmäßig wiederholten Markierungen, die das Ganze strukturlos erscheinen lassen, Verschiedenes, sondern auch der einzelne kann in den gleichen Vorgaben verschiedene Formen sehen und zwischen ihnen alternieren. Lichtenberg beschreibt damit etwas, das seit den 1830er Jahren Gegenstand wissenschaftlicher Wahrnehmungsphysiologie ist und im frühen 20. Jahrhundert in der Gestaltpsychologie eine wichtige Rolle spielt: das Wechseln des Aspekts bei der Perzeption. Insbesondere Umspringfiguren wie etwa der Necker-Würfel erlauben die Erfahrung, dass eine Linienfiguration einmal so, einmal anders gesehen werden kann. Das Musterbilden aus gleichmäßigen Markierungen, aus Strukturlosem, Dividuellem geht darüber hinaus, da es mehr als zwei inverse Möglichkeiten des Sehens zulässt, aber das Prinzip, in Markierungen etwas, hineinzusehen' oder im Gegenteil, nicht fähig zu sein, eine bestimmte Form in ihnen zu erkennen wie beim Rorschach-Test, teilen die verschiedenen Verfahren. Lichtenberg ist dem wahrnehmungspsychologischen Phänomen des Aspektsehens und Aspektwechsels immer wieder auf der Spur. Die Aufzeichnung hier erhält ihr Gewicht daraus, dass die Entdeckung zwar einem zufälligen Divertissement auf dem Krankenlager entstammt, aber Lichtenberg ihrer weit über die pleasures of boredom (GOMBRICH) hinausgehenden Bedeutung gewahr wird. Er entdeckt die formierende und transformierende Kraft des Sehens, und er hält sie nicht für eine nebensächliche, wenn auch spielerisch oder pädagogisch zu nutzende Störung, sondern für einen Teil der normalen Wahrnehmungstätigkeit.

Gedanken in dieser Richtung, zu Gesetzmäßigkeit und Zufall und zu deren Gegebenheit und Hervorbringung, hätten sich auch in Projekten gefunden wie: „Vielleicht die Beschreibung eines Dintenflecks” oder: „Theorie der Falten in einem Kopfkissen." ${ }^{14}$ Sie wurden nicht ausgeführt, aber sie böten einiges zu Fragen ,visueller Kultur': ungewöhnliche Ekphrasis, Klecksographologie, neue Seiten klassizistischer Ästhetik, pathognomische Linientheorie, Fraktale avant la lettre, wer weiß. Sie böten des weiteren einiges zur Frage nach der Zone von Wissen und Nichtwissen, Theorie und Nicht-Theoriefähigem, Methode und Zufall, aus der im wissenschaftlichen Arbeiten ebenso wie im Schreiben Neues hervorgeht. Und sie lieferten zusätzliche Facetten zum Bild eines Subjekts, das sich nicht aus der konstitutiven Macht des Denkens verstehen läßt.

\footnotetext{
${ }^{14}$ E 346, SB I, 421, und L 476, SB I, 919.
} 


\section{Mainberger S. - Versuchsanordnung öffentlicher Platz}

„Es denkt, sollte man sagen, so wie man sagt: es blitzt", ${ }^{15}$ ist ein zu Recht berühmt gewordenes Diktum. Nietzsche wird es aufgreifen und noch verschärfen, Ernst Mach, Freud, Musil werden es zitieren oder Ähnliches aufrufen. ,Es blitzt' figuriert nicht nur ein unfaßbar anonymes, dabei mächtiges und gefährliches Subjekt, sondern - anders als ,es regnet ${ }^{6}$ - auch die spezifische Zeitlichkeit eines Denkens, das nicht als Zustand oder Tätigkeit, sondern als Ereignis, Anfang, Sprung aus dem Gleis gefaßt wird. Dass es plötzlich geschieht, heißt aber nicht, es sei jenseits des Begreiflichen. Sein Entstehen zu studieren, seine Kräfte zu kennen und seine Potentiale $\mathrm{zu}$ nutzen, ist vielmehr eine ebensolche Herausforderung wie der kontrollierende Umgang mit dem entsprechenden physikalischen Phänomen. Versuche mit der Elektrizität und Versuche mit dem Gedankenblitz sind bei Lichtenberg parallele und einander zuweilen begegnende Unternehmungen; er selbst, seine Zeitgenossen und die Lichtenberg-Forschung haben das vielfach unterstrichen. Und die Beziehung geht über Analogien hinaus; denn die damalige Experimentierpraxis hat ihre theatrale und dramatische Seite in ihren Vorführungen, und gerade die Behandlung eines Themas wie das der Elektrizität ist mitnichten frei von Metaphern und Imaginationen. Insofern stellt sich das Verhältnis zwischen Wissenschaft und Schriftstellerei nicht nur einseitig dar, sondern vielleicht am besten in einer Elektrizitäts-Metapher ausgedrückt - als Wechselstrom.

Neben der Semantik liefert die grammatische Struktur des ,es blitzt ${ }^{`}$ Lichtenberg die Alternative zum cartesischen Modell. ${ }^{16}$ Statt des souveränen ,Ich` agieren bei ihm immer wieder andere Instanzen: die Materialität der Sprache etwa oder die Visualität des Schreibens, und aus ihnen gehen Gedanken hervor; das ungerichtet nach allen möglichen Seiten sich bewegende Sudeln hat allem voran den Zweck, genügend Ungenormtes und Unreines zur Verfügung zu stellen, das die Genese von Neuem ermöglicht.

Dieses Denken des ,es‘ - ein genitivus subjectivus - bringt keine unbezweifelbare Theorie hervor, sondern ist eine vielfältige, unvorhersehbare Praxis; sie lässt sich nicht positivieren. Ihr entspricht die bekannte Apologie der Zickzacklinie, ${ }^{17}$ der Lichtenberg

\footnotetext{
${ }^{15}$ K 76, SB II, 412.

${ }^{16}$ Auch Gedanken wie „Non cogitant, ergo non sunt” (J 379; SB I, 708) und der immer wieder beschworene aufklärerische Topos des ,Selbstdenkens' relativieren das nicht. Lichtenbergs Selbstbeobachtungen rütteln an den scheinbar festen Instanzen wie Ich und Wille; sein Subjekt ist weder das Descartes' noch dasjenige Kants, wenn das sensualistisch-skeptizistisch vielfältige und unfeste freilich auch nicht dasjenige Freuds, geschweige jüngerer Theoretisierungen ist.

${ }^{17}$ B 131, SB I, 82; außerdem F 995, SB I, 603; SB III, 865; vgl. auch die Zeichnung in SB IV, 852, u.a.
} 
Mainberger S. - Versuchsanordnung öffentlicher Platz

programmatischen Wert in seinen poetologischen Überlegungen verleiht. Sie ist indes keine Doktrin, sondern nur eine momentane Stabilisierung in einer Bildvorstellung, eine nur transitorische Formel. Denn wenn sich die von ihr ikonisierte Textbewegung, wie in der damaligen Sterne-Mode, als Masche und Manier etabliert, verfällt sie der Kritik, ${ }^{18}$ und dann bedarf auf einmal wieder die Gerade einer Apologie und einer ausdrücklichen Poetik. Die Gerade ist daher nicht mehr wie bei Sterne die Linie der ironisierten Moralität und der narrativen Teleologie, sondern die Schreiblinie vorbildlicher wissenschaftlicher und philosophischer Prosa; sie folgt gewissermaßen den Gesetzen der euklidischen Mathematik und der Schwerkraft, wie sie dem gut begründeten Gedanken eignet. Leibniz, Locke, Hartley sagen in diesem Sinn, was sie zu sagen haben, „dünne”, d.h. ohne das Brausen und Schäumen und den Wellengang der angemaßten Genies. ${ }^{19}$

Dieses Lob der Geraden als einer ,unrhetorischen', Figuren vermeidenden Rhetorik ergänzt die Hochschätzung der prima vista komplementär erscheinenden Metapher: Sie sorgt für oblique, die Bedeutung wendende Beziehungen. Hier gilt sie indes nicht als Umweg und Abweichung vom (normativen) Literalsinn, sondern im Gegenteil zur Konvention, zum Gelernten, wieder mathematisch gedacht, als die kürzeste Verbindung zwischen zwei Punkten: „Wenn man ein altes Wort gebraucht, so geht es oft in dem Kanal nach dem Verstand den das ABC-Buch gegraben hat, eine Metapher macht sich einen neuen, und schlägt grad durch. (Nutzen der Metaphern)". ${ }^{20}$ Auch ein Kanal ist ein Gleis: Und auch die Metapher, nicht nur die geometrisierende Vernunft, vermag eine Ecke zu schneiden - nämlich dann, wenn sie kein Topos ist, sondern ein Produkt des (kombinatorischen) Witzes, ein concetto, eine semantisch intrikate, epistemisch aber ,gerade', direkte, insofern ,einfache' Beziehung. Im Entdecken von Ähnlichkeit - seit Aristoteles ist das die kognitive Leistung der Metapher -, und dies entgegen den institutionalisierten und konventionellen Beziehungen zwischen den Dingen, quer zu den eingefahrenen Wegen, liegt die Kraft des denkenden ,es‘.

3) Diese Poetik kommt nicht aus ohne eine ihr entsprechende Lektüre. Ihre Rolle hält ein weiteres Linienbild fest. Es hat weniger Prominenz erlangt als die Zickzacklinie, aber es ist

\footnotetext{
${ }^{18}$ Vgl. D 610, SB I, 324.

${ }^{19} \mathrm{Vgl}$. E 501, SB I, 448.

${ }^{20} \mathrm{~F} 116$, SB I, 477.
} 
Mainberger S. - Versuchsanordnung öffentlicher Platz

nicht minder aussagekräftig; Lichtenberg widmet auch dieser Figur Elogen und verleiht ihr poetologische Signifikanz. Gemeint ist die durchbrochene, punktierte Linie. ${ }^{21}$ Sie markiert die Kunst, weniger zu sagen, als man könnte, und das Vertrauen auf einen mündigen Leser. Ein solcher mag Ausgespartes ergänzen und von einem Punkt zum nächsten springen - sozusagen von einer trockenen Stelle im Morast zu einer anderen; er braucht kein durchgezogenes Gleis. Warum aber, so mag man fragen, soll er, wo Lücken sind, nur von einem Punkt ,zum nächsten` springen? Eine Lücke lässt potentiell alle Richtungen zu - z.B. dann, wenn es auf die Schuhe nicht ankommt. Daher lassen sich Konjekturen nur in Grenzen kalkulieren; Ellipsen und Aposiopesen entziehen sich auktorialen Kontrollansprüchen, Gesprächsverläufe sind, wenn tatsächlich beide Seiten zum Zuge kommen, unabsehbar. Die punktierte Linie ist die einer liberalen Kommunikation, und das heißt auch einer Kommunikation mit Risiken. Sie lässt Freiheit zu, aber sie ist auch das Einfallstor für Kontingenz. Der Betrachter oder Leser wird demnach bei Lichtenberg auf doppelte, gegenläufige Weise aktiv: Wo kein Gleis ist, sorgt er für eines, und wo eines ist, schafft er die Möglichkeit, es zu verlassen ...

Auch das Modell für den selbstdenkenden Leser findet sich in der Szene mit dem Fenster auf den Platz: Die Beobachtung der ihn überquerenden Passanten mag sich an übliche wissenschaftliche Verfahren halten, aber diese werden auf einen nicht zum Metier des Physikers gehörigen Gegenstand angewandt. Das ist zweifellos ein Beispiel für disziplinäre Heterodoxie. Die Gehenden demonstrieren unter diesem Blick sichtbar, dass (im ersten Fall, im Zustand nach dem Regen) lebenspraktische Zusammenhänge Rationalität modifizieren und sie sich erst vollständig zur Geltung bringt, wenn Wissen, äußere Umstände und soziale Komponenten zusammenpassen; zugleich demonstrieren sie, dass Rationalität in der Praxis ein kollektiver Prozeß ist. Im Verhältnis zu logifizierendem Perfektionismus und Vernunftsolipsismus ist das eine Aufklärung über Aufklärung.

Der Leser nun befindet sich zu dem ihm vorgeführten Versuch und seinem Beobachter in der Position des Beobachters zweiter Stufe. Ihm zeigen sich - wie jenem am Fenster die Bahnen der Gehenden - die Denkbahnen eines Experimentalphysikers, aber nicht nur diese. Denn er hat angesichts der Sudelbücher - diesen Spuren intellektueller Raumdurchquerungen

\footnotetext{
21 Sie ist das Kennzeichen guter Schriftstellerei, vgl. B 86, SB I, 70. - Musil bezieht das Bild einer diskontinuierlichen Folge auf das Denken und unsere Vorstellung davon und bemüht seinerseits einen zeittypischen Vergleich mit einer technischen Aufzeichnungsart und der entsprechenden Wahrnehmung: „Wir denken überhaupt nicht discursiv, sondern sprungweise. Die Täuschung ist dieselbe wie bei einem Kinematographen.“ (MUSIL 1983: I, 117)
} 
Mainberger S. - Versuchsanordnung öffentlicher Platz

- den Vorteil, dass er sich die Versuchsanordnung nicht wie jener erst nach dem Muster entwerfen muss: „Man denke sich ein weißes Rechteck_..” Die Notate nötigen ihn vielmehr dazu, mit diesem Konstruktionsschema zu brechen und sich in eine nicht kodifizierte Praxis des Denkens involvieren zu lassen. Der Leser kann gar nicht umhin, den Gegenstand nicht nur $\mathrm{zu}$ sehen, sondern auch an seiner Erzeugung mitzuwirken und eine naive Auffassung, die Rezeption für etwas Passives hält, aufzugeben. Mit dem Bild der Platzüberquerung gesagt: Der Leser folgt den ,Punkten“, den diskontinuierlichen Aufzeichnungen, die der Schreibende im „Parallelogramm[]” der zu beschreibenden Blätter hinterlassen hat. Die Notate bilden durchbrochene Linien, die kreuz und quer verlaufen und Lese- und Denk-Bewegungen in alle möglichen Richtungen erlauben, oder sie sind Punkte, aus denen der Leser - Muster sehend, Ordnungen schaffend - Linien erzeugt. Selbst wenn er an seinem Fenster, dem aufgeschlagenen Buch, sitzen bleibt, beobachtet er also nicht nur, sondern er zieht auch die Linien aus. Diese krummen und geraden Gänge über einen weiten Platz, die aphoristischen Denkbahnen, sind wie die vielen geschnittenen Ecken: Der Witz schafft - außerhalb der Gleise und gegen alle Widrigkeiten - den knappsten Weg durch den Morast des Gedankenlosen. Dieser Weg entspricht nicht der geometrischen Linie, die überall die gleiche ist, sondern der situativ, von Fall zu Fall möglichen größten Ökonomie. Wo indes ein Weg der Nachahmung und Tradition als zufällige krumme Linie der Vorgänger verläuft, könnte sich das Denken seine eigene, abweichende Spur suchen. Voraussetzung dafür ist der gelegentliche Schneefall, d.h. plötzliche Verwirrung, Orientierungslosigkeit, Verschwinden der Bahnen, kurz: ein Einbruch des Nicht-Wissens. Die Passanten in Lichtenbergs Szenario suchen diesem Zustand so schnell wie möglich $\mathrm{zu}$ entgehen, für den Beobachter und Sudelbuchschreiber aber ist dergleichen das unerhörte Geschenk eines Settings zur experimentellen Betätigung.

\section{Literaturverzeichnis}

ARBURG, Hans-Georg von. Kunst-Wissenschaft um 1800. Studien zu Georg Christoph Lichtenbergs Hogarth-Kommentaren. Göttingen, Wallstein Verlag, 1998.

Busch, Werner. Das sentimentalische Bild. Die Krise der Kunst im 18. Jahrhundert und die Geburt der Moderne. München, C.H. Beck, 1993. 
Mainberger S. - Versuchsanordnung öffentlicher Platz

GAMPER, Michael. Fiktionen und Experimente. Lichtenberg und die Elektrizität. In: „Es ist nun einmal zum Versuch gekommen". Experiment und Literatur I 1580-1790. Hg. von Michael Gamper, Martina Wernli, Jörg Zimmer, Göttingen, Wallstein Verlag, 2009, 359-389.

Hankins, Thomas L. Science and the Enlightenment. Cambridge, London etc., Cambridge University Press, 1985.

LEBENSZTEJN, Jean-Claude. L'art de la tache. Introduction à la ,Nouvelle methode' d' Alexander Cozens. Montélimar, Éditions du Limon, 1990.

Lichtenberg, Georg Christoph. Schriften und Briefe. Hg. von Wolfgang Promies. 4 Bde. und 2 Kommentarbde. Frankfurt am Main, Zweitausendeins, 1994. (SB)

MAINBERGER, Sabine. Einfach (und) verwickelt. Zu Schillers ,Linienästhetik'. Mit einem Exkurs zum Tanz in Hogarths Analysis of Beauty. In: Deutsche Vierteljahrsschrift für Literaturwissenschaft und Geistesgeschichte 79(2), 2005, 196-251.

MusiL, Robert. Tagebücher. Hg. von Adolf Frisé. Neu durchges. u. erg. Auflage. 2 Bde. Reinbek b. Hbg, Rowohlt, 1983. 